International Journal of Wireless \& Mobile Networks (IJWMN) Vol. 5, No. 3, June 2013

\title{
BER Performance of Antenna Array-Based Receiver using Multi-user Detection in a Multipath Channel
}

\author{
Rim Haddad \\ Laboratory research in telecom systems 6'Tel@ SUP'COM \\ High School of Communicationof Tunis \\ Route de Raoued, Km 3,5 2083 Ariana, Tunisia \\ rim.haddad@yahoo.ca
}

\begin{abstract}
Antenna promises to provide significant increases in system capacity and performance in wireless systems. In this paper, a simplified, near-optimum array receiver is proposed, which is based on the angular gain of the spatial filter. This detection is then analyzed by calculating the exact error probability. The proposed model confirms the benefits of adaptive antennas in reducing the overall interference level (intercell/intracell) and to find an accurate approximation of the error probability. We extend the method that has been proposed for propagation over Nakagami-m fading channels, the model shows good agreements with simulation results.
\end{abstract}

Keywords: MAI, MUD, BER, Nakagami fading.

\section{Introduction}

Several smart antenna systems have been proposed, and demonstrated at the base station (BS) of the wireless communication system, and these have shown that significant increases in capacity are possible [1],[2].

In principle, a MUD receiver allows constructive combination of multi-path signals received by an array of antennas while minimizing the MAI's contribution. Besides, providing the average error probability for K users with DOA's (Direction of Arrival) uniformly distributed within a symmetric support around the array broadside has been derived for chip and phase asynchronous DS-CDMA system [3],[4].

The Bit Error Rate (BER) is considered to be one of the most important performance measures for communication systems and hence it has been extensively studied. The exact analytical evaluation of the probability of error in DS-CDMA system, is still an open subject.

Gaussian approximations of the Multiuser Interference (MUI) are used to reduce the problem, and to be tractable namely when the average performance is of interest.

However, the accuracy of the Gaussian approximation technique depends on the specific configuration of the system.

When analyzing the BER performance, the interference sources, namely the Multiple Access Interference (MAI) are commonly assumed to be Gaussian distributed, for coherent receivers over Nakagami-m fading channels [5], and for linear Minimum Mean Square Error (MMSE)MUD. 
Hence in this paper, we will derive an accurate BER formula for Nakagami - faded DS-CDMA in the context of asynchronous transmission and we proposed to adapt the Gaussian approximations to antenna array systems by properly accounting for the noise and the MUI after beamforming. This is carried out by considering an approximation of the angular gain in smart antenna array systems.

In this paper, we propose a novel approach to evaluate the average probability of error by considering an approximation of the spatial filter. The angular gain function is approximated by a fixed beamwidth $\theta_{B W}$, for the passband and by an attenuation $\alpha_{0}$. For the user of interest, all the remaining $k-1$ interferers are partitioned into in-beam / out-beam MUI. The analytical formulas ofthe average error probability is counted differently for the in-beam/out-beam interferers and we validate our research for single antenna systems.

We organize the rest of the paper as follows. In section 2 we introduce our system model, followed by the array gain approximation in section 3. The average probabilityof error with adaptive antenna and computation results are provided in section 4 and section 5 respectively. We conclude in section 6 .

\section{System Model}

\subsection{Signal Model}

The base station is equivalent with a uniform linear array (ULA) of M equi-spaced identical elements.

The array receives the signals from the $\mathrm{K}$ subscribers located in the far field zone of the array. We assume that all the signals are uncorrelated and each user transmits a binary phase-shift keying (BPSK) symbols.

The base band equivalent model is considered for asynchronous modulation waveforms $S_{1}(t), S_{2}(t), \ldots S_{K}(t)$. The transmitted signal of the $K^{t h}$ user is:

$$
x_{k}(t)=>_{i} b_{k}^{i} S_{k}(t-i T)
$$

$b_{k}^{i} \in\{-1,+1\}$ is the $i^{\text {th }}$ transmitted BPSK symbol and Tis the symbol interval.The user's signal $x_{k}(t)$ propagates through a multipath channel, $\theta_{k}$ is the DoA of the $k^{t h}$ user.The impulse response can be written as:

$$
h_{k}(t)=\sum_{\underline{m=1}}^{L} \alpha_{k, m} \delta\left(t-\tau_{k, m}\right)
$$

Wherein $\alpha_{k, m}$ and $\tau_{k, m}$ are the complex gain and delay of the $m^{\text {th }}$ path. We assume thatall the users have the same number of paths $L$, the delay $\tau_{k, m} \in[0, T[$ have increasing values: $0 \leq$ $\tau_{k, 1} \leq \tau_{k, 2} \leq \cdots \leq \tau_{k, L}<T$, for $\forall k$

The spatial response of the array due to an incident plane wave from $\theta_{k}$ direction is modeled by the array steering vector $a\left(\theta_{k}\right)$ [6], [7]. Wherein $a\left(\theta_{k}\right)$ is the $M \times 1$ vector that describes the array response to the $\operatorname{DoA} \theta_{k}$, and the $n^{\text {th }}$ element for a linear of half-wavelength spaced antennas is:

$$
\left[a\left(\theta_{k}\right)\right]_{n}=\exp \left(-j(n-1) \pi \sin \theta_{k}\right) .
$$


At the receivers, the $M \times 1$ vector of that received signal for the $k^{\text {th }}$ user.

$$
\begin{gathered}
r_{k}(t)=a\left(\theta_{k}\right) h_{k}(t) * x_{k}(t) . \\
\left.r_{k}(t)=a\left(\theta_{k}\right)\right\rangle_{\bar{i}} b_{k}^{i} \sum_{m=1}^{L} \alpha_{k, m} S_{k}\left(t-i T-\tau_{k, m}\right) .
\end{gathered}
$$

The received signal of the $K$ users' signal can be written as:

$$
r(t)=\sum_{k=1}^{K} r_{k}(t)+\sigma n(t) .
$$

The noise $n(t)$ is assumed to be a zero-mean temporally and spatially uncorrelated Gaussian process, with $E\left[n . n^{*}\right]=I \cdot \delta(\tau), \sigma^{2}$ is the power of the AWGN. We assume that the spatial correlation of noise arising from intercell interference is not considered.

After the beamformingwith the $M \times 1$ spatial filter $w_{l}$ for the $l^{t h}$ user, the output of the $l^{\text {th }}$ filter matched to $S_{l}(t)$ is:

$$
\begin{gathered}
y_{n^{\prime \prime} l}[j]=\mid S_{l}^{*}\left(t-\tau_{l, n}-j T\right) r(t) d t . \\
\left.y_{n^{\prime \prime} l}[j]=\sum_{k=1}^{K}\right\rangle_{\bar{i}} b_{k}^{i} G\left(\theta_{k / l}\right) \sum_{\bar{m}=1}^{L} \alpha_{k, m} \rho_{k, l}\left((i-j) T+\tau_{l, n}-\tau_{k, m}\right)+\sigma n_{n^{\prime} l}^{\prime}[j] .
\end{gathered}
$$

$G\left(\theta_{k / l}\right)=w_{l}^{H} \cdot a\left(\theta_{k}\right)$, where $w_{l}^{H}$ is the weight vector and $\mathrm{H}$ denotes Hermitian transpose: is the spatial gain of the beamformer designed for the angle $\theta_{l}$. And $\rho_{k, l}(\tau)=\int S_{k}(t+\tau) S_{l}^{*}(t) d t$ is the cross correlation function between signatures.

The matched filter output contains the self interferenceISI and the MUI. The self interference ISI is written by this equation:

$$
y_{n / l}^{I S I}(j)=G\left(\theta_{l / l}\right) \sum_{i} b_{l}^{i} \sum_{m=1}^{L} \alpha_{l, m} \rho_{l, l}\left((i-j) T+\tau_{l, n}-\tau_{l, m}\right) .
$$

The MUI is:

$$
y_{n / l}^{M U I}(j)=\sum_{k=1, k \neq l}^{K} \sum_{i} b_{k}^{i} G\left(\theta_{k / l}\right) \sum_{m=1}^{L} \alpha_{k, m} \rho_{k, l}\left((i-j) T+\tau_{l, n}-\tau_{k, m}\right) .
$$

The noise power after the Beamforming is $\sigma^{2 /} M$. To make the analytic evaluation of error probability computation feasible, we have to assume that the waveforms are randomly generated on each BPSK symbol with outcome uniform on $\{-1,+1\}$.

\subsection{MUD Receivers}

The signals from different antenna elements are weighted and "summed" to "optimize" the quality of the signal [8] (Fig. 1).

In a multipath environment, the MUD receiver employs the maximal ratio combining by filtering each path of each user's signal with the corresponding space filter and then combining the match filter outputs[9],[10]. 


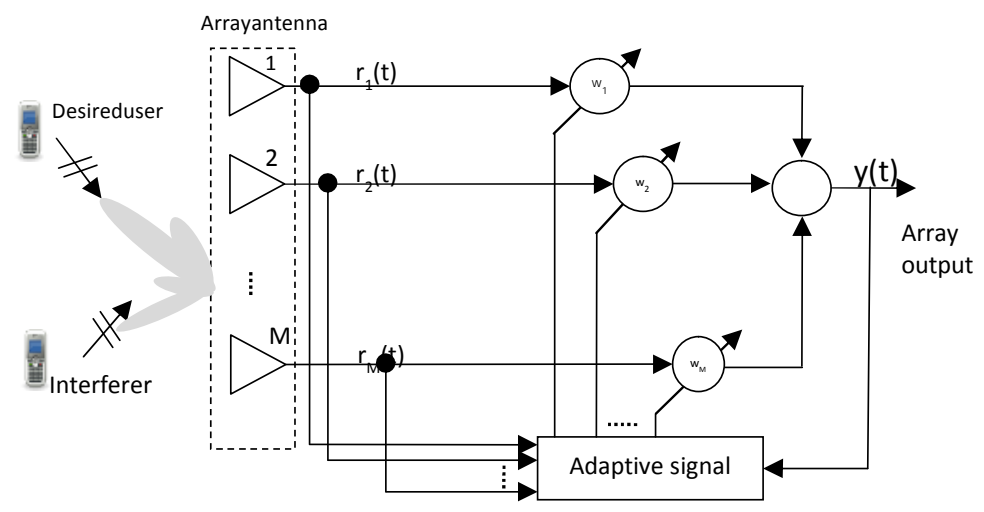

Fig.1.Block diagram of a smart antenna system.

All the paths experience the same level of noise and interference. The decision variable after the beamforming is assumed to be obtained by the maximal ratio combining.

$$
y_{l}[j]=\sum_{m=1}^{L} \alpha_{l, m} y_{m^{\prime} l}[j] .
$$

The use of beamforming in MUD receivers is expected to be effective mainly in reducing intercell interference. However, to evaluate the advantage of the array processing in reduction of intercell interference a simplified model of CDMA system can be viewed as a synchronous model with an increased number of fictitious users. Therefore, the synchronous model CDMA for one path, $\tau_{1,1}=\tau_{2,1}=. . \tau_{K, 1}=0$ for $\forall k$, is considered here as a useful example to gain insights on the array processing gain in MUD [10].

The received CDMA signal after spatial filtering and dispreading is:

$$
y_{l}[j]=\sum_{k=1}^{K} \sum_{j} b_{k}^{j} \alpha_{k, 1} G\left(\theta_{k / l}\right) \rho_{k, l}+\sigma n_{l}^{\prime}[j] ; \text { for }=1,2, \ldots, K .
$$

where $\rho_{k, l}=\rho_{k, l}\left(\tau_{k, 1}=0\right)$.

\subsection{Channel Model}

The delays $\tau_{k, m}$ and phases $\alpha_{k, m}$ are i.i.d random variables uniformly distributed.The used model for frequency selective multipath channel for mobile communication is the Nakagami model, the amplitudes $\left|\alpha_{k, m}\right|$ are independent random variables with Nakagami probability density function.

$F\left(\left|\alpha_{k, m}\right|, \xi, \sigma_{k, m}^{2}\right)$ where:

$$
F\left(\left|\alpha_{k, m}\right|, \xi, \sigma_{k, m}^{2}\right)=\frac{2 \xi^{\xi}\left|\alpha_{k, m}\right|^{2 \xi-1}}{\sigma_{k, m}^{2 \xi} \Gamma(\xi)} \exp \left(-\frac{\xi\left|\alpha_{k, m}\right|^{2}}{\sigma_{k, m}^{2}}\right) .
$$

$\Gamma(\bullet)$ is the gamma function. The fading parameter $\xi(\xi \geq 1 / 2)$ spans different distributions: The Rayleigh distribution for $\xi=1$ while in the limit $\xi \rightarrow \infty$ the fading channel converges to a nofading channel.

To simplify, we assume that the power delay profile of the path strengths is the same for all the users:

$$
\sigma_{k, m}^{2}=\sigma_{k, 0}^{2} \exp (-\delta(m-1))
$$

The parameter $\delta, \delta \geq 0$ is the decay rate, the total average fading power. 


$$
\sigma_{k}^{2}(\delta)=\sum_{m=1}^{L} \sigma_{k, m}^{2}=\sigma_{k, 0}^{2} q(L, \delta) .
$$

It depends on the decay rate and the number of paths: $q(L, \delta)=L[1-\exp (-\delta L)][1-\exp (-\delta)]$. For $\delta=0$ the power delay profile is uniform and $q(L, \delta)=L$.

\section{Array Gain Approximation}

For the user of interest (say $\theta_{1}$ ) conventional beamforming weights are considered $w_{1}=$ $a\left(\theta_{1}\right) / M$, the angular gain function for conventional beamforming is $\left.G\left(\theta ; \theta_{1}\right)=a\left(\theta_{1}\right)^{H} a(\theta)\right)^{\prime} M$.

To simplify the computations in the following, the gain $\left|G\left(\theta^{\prime} \theta_{1}\right)\right|^{2}$ can be approximated by a piece-line function $\left|G_{e q}\left(\theta^{\prime} \theta_{1}\right)\right|^{2}$ that models the pass-band or in-beam with support $f_{A O A}\left(\theta_{1}\right)=$ $\left[\theta_{1}-\theta_{B W}\left(\theta_{1}\right), \theta_{1}+\theta_{B W}\left(\theta_{1}\right)\right]$ with a linear gain and the out-beam (with support $\bar{f}_{D O A}\left(\theta_{1}\right)$ with an equivalent attenuation $\alpha_{0}$ ) [11].

The gain can be approximated by:

$$
\left|G_{e q}\left(\theta / \theta_{1}\right)\right|^{2}=\left\{\begin{array}{cc}
1-\frac{1}{2} \frac{\left|\theta-\theta_{1}\right|}{\theta_{B W}\left(\theta_{1}\right)} & \text { for } \quad \theta \in f_{D O A}\left(\theta_{1}\right) \\
\alpha_{0} & \text { for } \quad \theta \in \bar{f}_{D O A}\left(\theta_{1}\right)
\end{array}\right.
$$

The beamwidth $\theta_{B W}\left(\theta_{1}\right)$ depends on the number of antennas $\mathrm{M}$ and $\theta_{1}$. The support $\theta=$ $f_{D O A}\left(\theta_{1}\right) \cup \bar{f}_{D O A}\left(\theta_{1}\right)=\left[-\Delta \theta^{\prime} 2, \Delta \theta^{\prime} 2\right]$ covers all the admissible DoAs in a mobile system with three-cell sectorization the angles range in $\pm 60^{\circ}$.

For small deviations from the broadside (for $\theta_{1} \approx 0 \mathrm{deg}$ ), the beamwidth $\theta_{B W}$ optimized for $\theta_{1}=0$ can be transformed into the beamwidth for any value $\theta_{1}$ by [12]:

$$
\theta_{B W}\left(\theta_{1}\right)=\frac{\theta_{B W}}{\cos \theta_{1}}
$$

By using this model, the in-beam interferers can be easily evaluated for any DoA of interest $\theta_{1}$ as in those in the support $f_{D O A}\left(\theta_{1}\right)$. The approximation parameters are expected to overestimate the average BER when employing minimum variance beamforming with a small number of users $\mathrm{K}$.

\section{Error Probability with Adaptive Antenna}

\subsection{No-fading channels}

We consider a system with $L=1$ and $A=\left[\alpha_{1}, \alpha_{2}, \ldots, \alpha_{K}\right]$, the error probability $P_{e}\left[A^{2}, \sigma^{2} \prime^{\prime} M, K_{I}\right]=P\left[E^{\prime} A,\left|B\left(\theta_{1}\right)\right|\right]$ depends only on the number of in-beam interferers $K_{I}$ and not on their DoAs.

The average error probability reduces:

$$
(E)=\sum_{K_{I}=0}^{K-1} P_{e}\left[A^{2}, \frac{\sigma^{2}}{M}, K_{I}\right] \int_{-\Delta \theta / 2}^{\Delta \theta / 2} p\left(K_{I}\right) f_{\theta}\left(\theta_{1}\right) d \theta_{1} .
$$

$p\left(K_{I}\right)$ is the probability of having $K_{I}$ in-beam interferers. The probability of an In-beam interferer:

$$
p\left(\theta \in \theta\left(\theta_{1}\right)\right)=\int_{\theta_{1}-\theta_{B W}\left(\theta_{1}\right)}^{\theta_{1}+\theta_{B}\left(\theta_{1}\right)} f_{\theta}(\xi) d \xi .
$$

Depends on the beamwidth $\theta_{B W}\left(\theta_{1}\right)=\theta_{B W^{\prime}} \cos \theta_{1}$. For DOAs uniformly distributed within the support $\left[-\Delta \theta^{\prime} 2,+\Delta \theta^{\prime} 2\right]$, the probability (19) depends on the beamwidth $\theta_{B W}\left(\theta_{1}\right) \operatorname{compared}$ to the overall support $\theta$. 


$$
p\left(\theta \in \theta_{1}\right)=\frac{2 \theta_{B W}\left(\theta_{1}\right)}{\Delta \theta}=\frac{\eta}{\cos \theta_{1}}
$$

Where $\eta=\frac{2 \theta_{B W}}{\Delta \theta}$ depends on the beamforming criterion exploited.

The average BER (18) becomes:

$$
P(E)=\sum_{K_{I}=0}^{K-1} \eta^{K_{I}}\left(\begin{array}{c}
K-1 \\
K_{I}
\end{array}\right) \chi\left(\eta, K, K_{I}\right) P_{e}\left[A, \frac{\sigma^{2}}{M}, K_{I} \mid .\right.
$$

Where $\chi\left(\eta, K, K_{I}\right) \approx(1-\eta)^{K-K_{I}-1}$ for matched filter receiver (receiver with $L=1$ branch) the BER depends on the interference: [11]

$$
\left.P_{e}\left[A, \frac{\sigma^{2}}{M}, K_{I}\right]=Q \mid\left(\frac{\sigma^{2}}{M A^{2}}+\frac{\sigma_{I}^{2}\left(K_{I}\right)}{A^{2}}\right)^{-1 / 2}\right\rceil .
$$

$Q[\cdot]$ is the Gaussian Q-function.The average level of interference (for chip and phase asynchronous) [9]:

$$
\sigma_{I}^{2}\left(K_{I}\right)=\frac{3}{4} A^{2} \frac{K_{I}}{3 N}+\alpha_{0} A^{2} \frac{K-K_{I}-1}{3 N}
$$

Counted for the $K_{I}$ in-beam and the $\left(K-K_{I}-1\right)$ out-beam users. For small values of $\eta$, the number of in-beam interferers is small. The more accurate approximation in [13] could be used for receivers with adaptive antennas.

For MMSE-MUD receivers with adaptive antenna arrays, the BER depends only on the in-beam users as the attenuated out-beam users are approximately decoupled. The evaluation of BER for the user of interest [4]:

$$
P_{e}\left(A^{2}, \frac{\sigma^{2}}{M}, K_{I}\right)=Q\left|\left(\frac{M A^{2}}{\sigma^{2}}-\frac{\frac{M A^{2}}{\sigma^{2}} \rho^{2} \frac{3}{8} K_{I}}{1+\frac{\sigma^{2}}{M A^{2}}+\rho\left(\frac{3}{8} K_{I}-1\right)}\right)^{1 / 2}\right| .
$$

The Gaussian approximation is based on the asymptotic analysis for $K_{I} \rightarrow \infty$ is used to derive (24), it is accurate enough to yield meaningful conclusions in the evaluation of the benefits of the MMSE-MUD in a receiving system employing an adaptive array system, and it decouples the effects of the spreading signatures in the analysis.

The array gain $1 / M$ for the AWGN is included either in the BER for matched-filter receiver (22)and in MMSE-MUD (24). In order to compare the benefits of the spatial diversity for a varying number of antennas, the SNR is measured after the Beamforming as $M A^{2 /} 2 \sigma^{2}$.

The average BER for any arbitrary DoAs distribution is conceptually dependent on $p(\theta \in$ $\left.f_{D O A}\left(\theta_{1}\right)\right)$ to get $p\left(K_{I}\right)$ for each $\theta_{1}$ and then averaging with respect to $\theta_{1}(18)$. The DoA's distribution might become a design parameter.

This occurs when MAI reduction is obtained by dynamically assigning the radio resource according to their DoAs.

\subsection{Nagakami-m fading channels}

In this section, we extend the concepts discussed above to the Nakagami m-fading case. The average performance needs to be evaluated from (18) by averaging with the Pdf of fading. The error probability $P\left(E^{\prime} A, K_{I}\right)$ depends on the instantaneous fading SNR $\gamma\left(\alpha_{1}, K_{I}\right)$ and on the number of in-beam interferers $K_{I}$.

Under the Gaussian approximation, the SNR at the decision variable has been evaluated by Eng and Milsten [5] for propagation over Nakagami fading channels. 


$$
\gamma\left(\alpha_{1}, K_{I}\right)=\sum_{m=1}^{L}\left|\alpha_{1, m}\right|^{2} \times\left(\frac{\sigma^{2}}{2 M}+\sigma_{A}^{2} q(L, \delta) \frac{\frac{3}{4} K_{I}+\alpha_{0}\left(K-K_{I}-1\right)}{3 N}+\sigma_{A}^{2} \frac{q(L, \delta)-1}{2 N}\right)^{-1} .
$$

Let the number of resolvable multipaths of each user be $L$, for $L=1$ (no multipath), the last term in (25) vanishes as $q(L, \delta)=1$; if $\delta=0$ it is $q(L, \delta)=L$ and the overall level of MUI and ISI (self interference) increases according to the degree of time diversity: $L$.

The error probability depends on the desired user $\theta_{1}$ and the number of in-beam interferers $K_{I}$ is $P\left(E^{\prime} \theta_{1}, K_{I}\right)$ can be evaluated in term of effective SNR: [5]

$$
\bar{\gamma}\left(K_{I}\right)=\frac{q(L, 2 \delta)}{2 \xi q(L, \delta)} \times\left(\frac{\sigma^{2}}{2 \sigma_{A}^{2} M}+q(L, \delta) \frac{\frac{3}{4} K_{I}+\alpha_{0}\left(K-K_{I}-1\right)}{3 N}+\frac{q(L, \delta)-1}{2 N}\right)^{-1} .
$$

For the case of Rayleigh fading $(\xi=1)$ and flat delay profile $\delta=0$.

The equation $P\left(E^{\prime} \theta_{1}, K_{I}\right)=P_{e}\left(\sigma_{A}^{2}, \sigma^{2 /} M, K_{I}\right)$ :

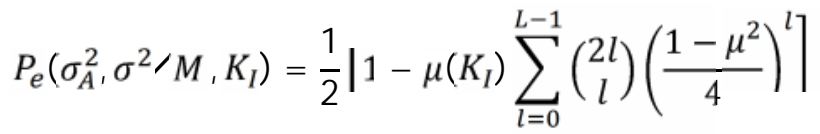

where:

$$
\mu\left(K_{I}\right)=\sqrt{\frac{\bar{\gamma}\left(K_{I}\right)}{1+\bar{\gamma}\left(K_{I}\right)}} .
$$

To evaluate the average BER, we sum over the cardinality of the in-beam set and average with respect to $\theta_{1}$ like the equation (24).

For uniformly distributed DoAs, it is:

$$
P(E)=\sum_{K_{I}=0}^{K-1} \eta^{K_{I}}\left(\begin{array}{c}
K-1 \\
K_{I}
\end{array}\right) \chi\left(\eta, K, K_{I}\right) P\left(\sigma_{A}^{2}, \sigma^{2 /} M, K_{I}\right) .
$$

which is dual equation of (21) for fading channels.

\section{Computation Results and Discussions}

In this section, we carry out the simulatedresults that have been obtained by applying a model of spatial filter that allows describing the angular gain function: the in-beam is approximated with a fixed beam-width and the out-beam with an equivalent attenuation.

The approximations used to evaluate the average performance for DS-CDMA receivers are validated here with numerical results. In the proceeding simulations, the following assumptions are made for all the users:

- The multipath channel parameters: number of paths $L$, DoAs $\theta_{k}$ are independent and uniformly distributed.

- All the users are received with the same average power as in a system with a perfect power control.

- The $M$ omni-directional antennas are arranged in a uniform linear array half wavelength spaced based on the conventional beamforming.

- The SNR is measured after the beamforming so that the 1/' $M$ array gain for AWGN is implicitly compensated to focus the attention on the gain arising from spatial diversity.

Fig. 2 illustrates the average probability of 2D-RAKE receivers versus SNR (solid line) for $L=1$ branch, $M=8$ antennas, $K=8$ and 16 users $(\mathrm{N}=31)$ for no-fading channel. 
Numerical experiments show that MUD receiver performance degrades down to single antenna receiver when the spatial filtering is in no way effective in reducing the MUI (for low SNR). Single user/single antenna (M=1) lower bound for AWGN (Fig.2) and Rayleigh fading channel (Fig.3). Simulation results are close to the analytical results proposed in this paper by accounting for the effects of the in-beam/out-beam interferers.Fig. 3 shows the average BER using the same parameters as in Fig. 2 in Rayleigh fading channel.

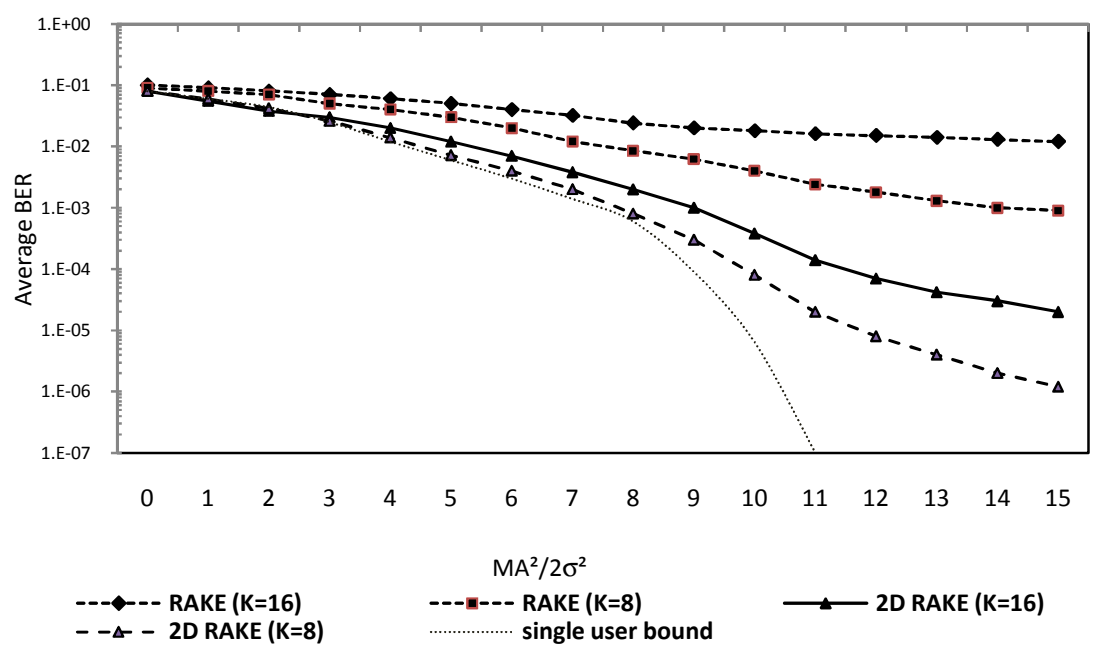

Fig. 2.Average BER versus $S N R$ for no fading channel for $L=1$ path, $M=8$ antennas, $K=8$ and 16 users.

Simulation results are close to the analytical results proposed in this paper by accounting for the effects of the in-beam / out-beam interferers.

Besides, the receivers based on the adaptive arrays demonstrate the efficacy in reducing the overall interference level.

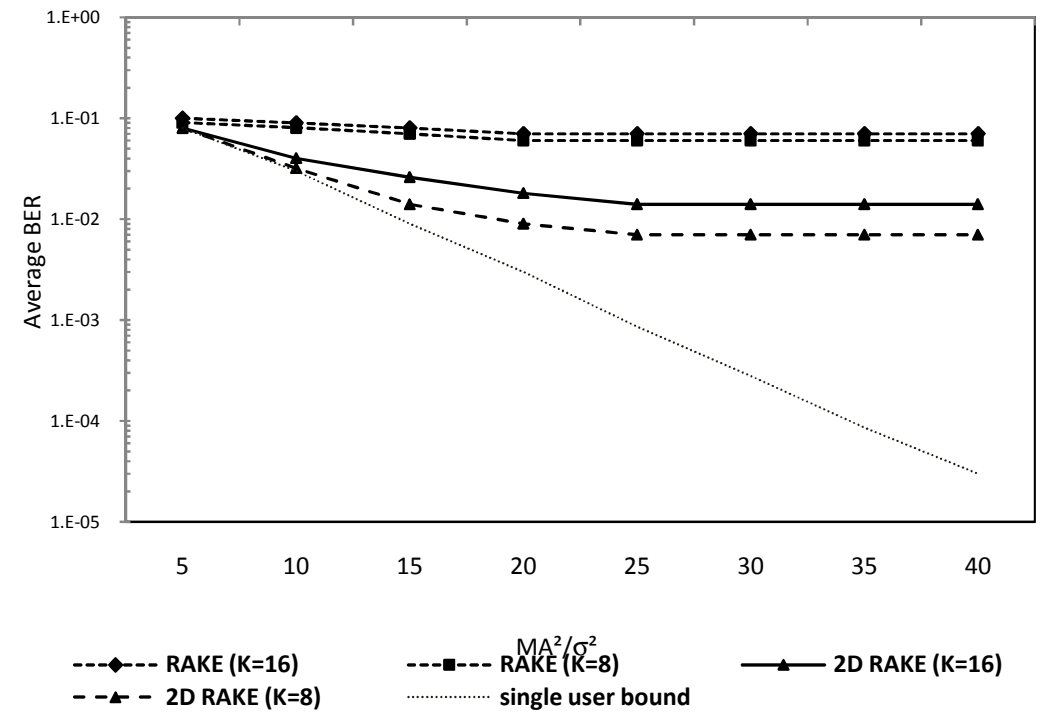

Fig. 3. Average BER versus $S N R$ in Rayleigh fading channel for $L=1$ path, $M=8$ antennas, $K=8$ and 16 users. 
International Journal of Wireless \& Mobile Networks (IJWMN) Vol. 5, No. 3, June 2013

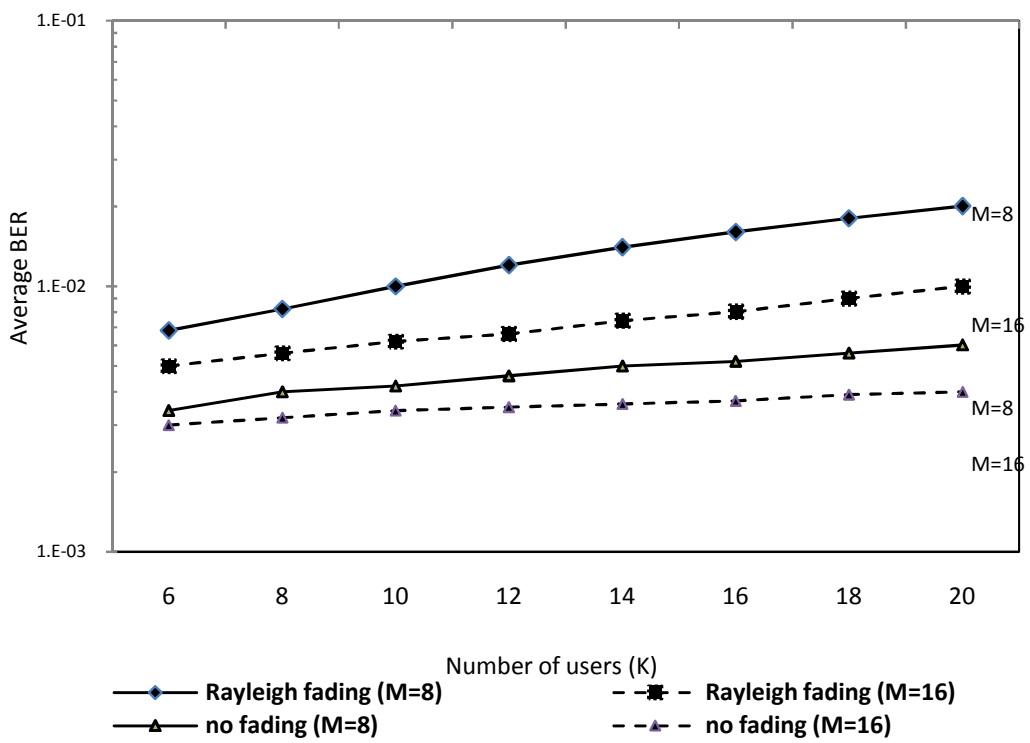

Fig. 4.Average BER versus the number of users $\mathrm{K}$ for no fading and Rayleigh fading channels for $\mathrm{L}=1$ path, $M=8$ and 16 antennas.

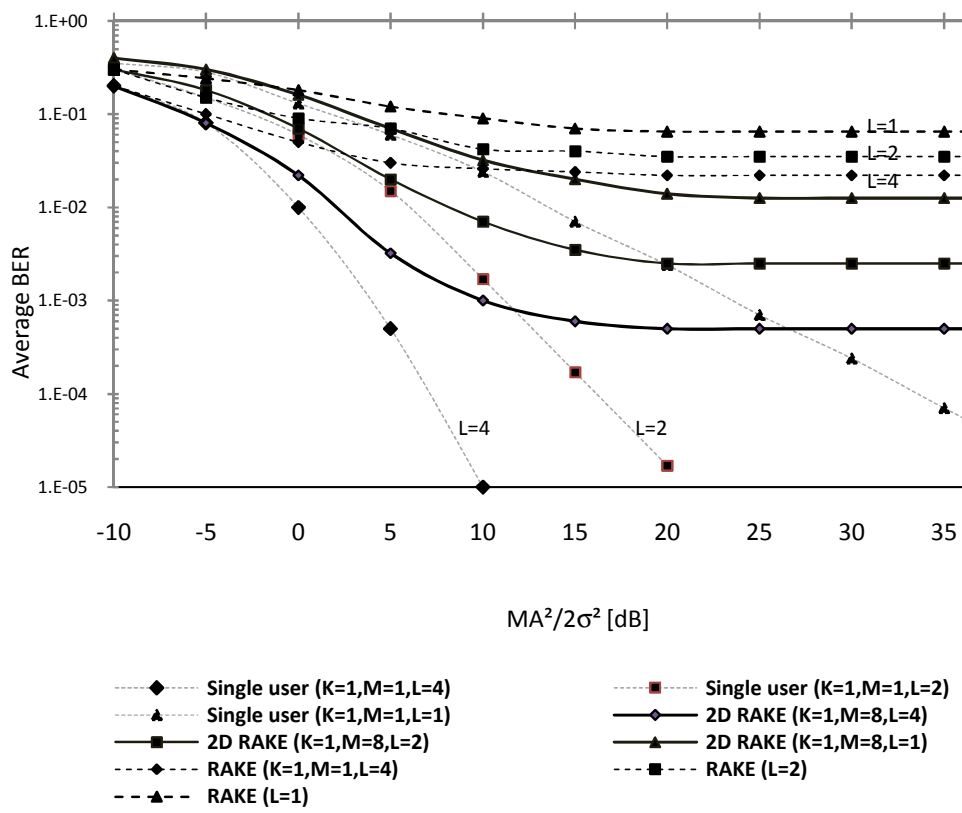

Fig. 5.Average BER versus $S N R$ for $2 D-R A K E ; M=8$ antennas and $K=16$, single antenna $(M=1)$ RAKE and single user/single antenna in multi-path Rayleigh fading channels ( $L=1,2,4)$.

The influence of the interference can be reduced by decreasing the probability of having an inbeam interferer $\eta$. This is illustrated in Fig.4, where the BER performance is shown by varying the number of users for no-fading and Rayleigh fading channels.

From Fig.4, it can be noticed that the same average BER can be obtained by doubling the number of antennas $M$ and the number of users $K$ either for no-fading or fading channels.

Therefore, as a rule, the average performance (or the level of the in-beam interference) remains the same as far as the ratio $M / K$ remains constant. This conclusion can be shown even when we neglect the influence of the out-beam interference. 
International Journal of Wireless \& Mobile Networks (IJWMN) Vol. 5, No. 3, June 2013

Fig.5 investigates the average BER for MUD receiverwith adaptive antennas for propagation over $\mathrm{L}$ paths frequency selective Rayleigh fading channel (for $\mathrm{L}=1,2,4)$ versus $\mathrm{SNR}(\mathrm{M}=8$, $\mathrm{K}=16, \mathrm{~N}=31$ ).

Fig.5 shows either for varying SNR $\left(S N R=M \sigma_{A}^{2}{ }^{\prime} \sigma^{2}\right)$ or increasing number of users, that multipath channels (large L) and angular diversity can improve satisfactory performance when exploited jointly.

\section{Conclusion}

The effects of the spatial filter for adaptive uniform linear arrays can be described by an equivalent model that is useful to evaluate the average interference and to find an accurate approximation of the average probability of error. We showed in simulations that we can influence in the number of antennas and users to evaluate BER. The BER is expected to fall well below the optimum when more number of antennas is used, but with a trade-off of increased cost and complexity. Besides, we noticed that the average performance (or the level of the in-beam interference) remains the same as far as the ratio $M^{\prime} K$ remains constant.In a continuation of the study, which we have already started, is to evaluate the average BER in forward link (base to mobiles) where each user experience the same temporal channel for all the received signals.

\section{References}

1. D. Piazza, N.J. Kirsh, A. Forenza, "Design and Evaluation of a reconfigurable antenna array for MIMO systems”, IEEE Transactions on Antennas and Propagation, vol.56, n`3, pp. 869-881, March 2008.

2. B.A. Bjerke, Z. Zvonar, and J.G. Proakis, "Antenna diversity combining aspects for WCDMA systems in fading multipath channels", IEEE Transactions on Wireless Communications, vol.3, $\mathrm{n}^{\circ} 1$, pp. 97-106, Jan 2004.

3. C.D. Iskander and P.T Mathiopoulos, "Performance of multicode DS/CDMA with M-array orthogonal modulation in multipath fading channels", IEEE Transactions on Wireless Communications, vol.3, n², pp.209-223 Jan. 2004.

4. S.Belfiore, "Smart Antenna Systems for mobile platforms", P.h.D Dissertation Arizona State University, Dec. 2002.

5. T. Eng and L.B. Milstein, "Coherent DS-CDMA performance in Nakagami multi-path fading", IEEE Trans. Commun. Vol.43, pp.1134-1143, Feb/March/Apr 1995.

6. Li Zhang, NingXie, "Non linear optimization for adaptive antenna array receivers with a small datarecord size", Wireless Communications \& Mobile Computing, vol.9, pp. 239-249, Feb. 2009.

7. H. J. Li and T. Y. Liu, "Comparison of beamforming techniques for W-CDMA communication systems”, IEEE Transactions on Vehicular Technology, vol.52, no.4, pp. 752-760, July 2003.

8. S. Choi, J. Choi, H.J. Im, and B.Choi, "A novel adaptive beamforming algorithm for antenna array CDMA systems with strong interferers", IEEE Transactions on Vehicular Technology, vol. 51, no. 5, pp. 808-816, Sept. 2002.

9. X. Wang and H.V.Poor, "Space-Time Multiuser Detection in Multi-path CDMA channel", IEEE Trans. on Comm. vol. 47, pp.185-190, Feb.1999.

10. B.H. Khalaj, A. Paulraj and T. Kailath, "2D RAKE receivers for CDMA cellular system” Proc. IEEE GLOBECOM conf., pp400-404, Dec. 1994.

11. U. Spagnolini, "A simplified model for probability of error in DS-CDMA systems with adaptive antenna arrays", in Proc. IEEE International Conference on Communications (ICC), June 2001, pp.2271-2275.

12. D. H. Johnson and D. E. Dudgeon, Array Signal Processing: Concepts and Techniques. Englewood Cliffs, NJ: Prentice-Hall, 1993.

13. J. Holtzman, "A simple accurate method to calculate spread-spectrum multiple access error probabilities", IEEE trans. on Comm., vol.40, pp.461-464, Mar. 1992 